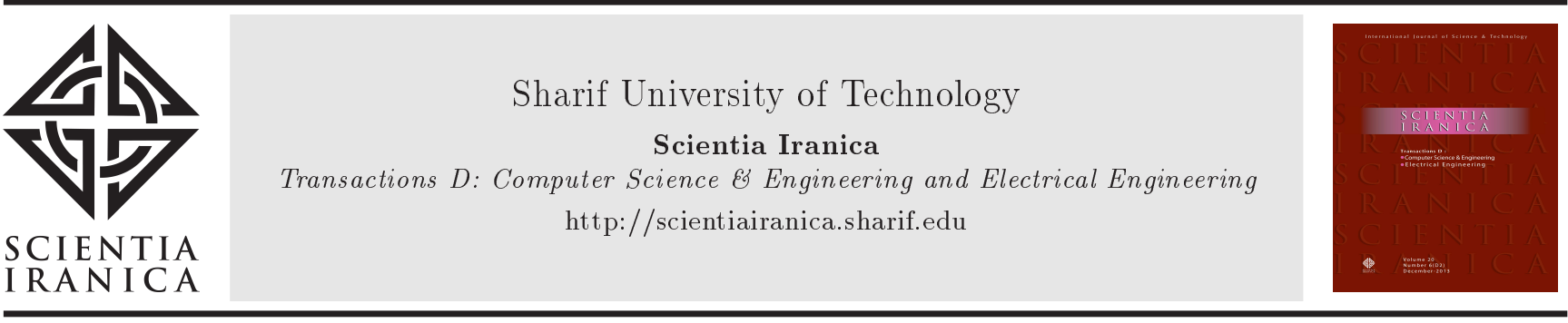

\title{
Enabling demand response potentials for resilient microgrid design
}

\author{
M. Chegnizadeh and A. Safdarian* \\ Department of Electrical Engineering, Sharif University of Technology, Tehran, Iran.
}

Received 13 August 2019; accepted 7 October 2019

\section{KEYWORDS}

Benders

decomposition;

Demand response;

Island operation;

Microgrid (MG);

Resiliency;

Time-varying price.

\begin{abstract}
The future microgrids (MGs) hosting a multitude of uncertain and intermittent local renewable generation resources are anticipated needing fast and flexible units on the generation side. However, demand response, as a load shaping tool, can alleviate this need. This paper proposes a model to consider demand response potential incorporated by timevarying prices in MG design studies. The model aims at maximizing profit of the MG owner while technical limits and constraints are adhered to. It also ensures that the designed MG is resilient to islanding events. To handle complexity of the model, Benders decomposition was used to decompose it into a master problem and two types of sub-problems. The master problem optimized binary variables indicating installing status of generating units and batteries. The first type of sub-problems optimized continuous variables and the second ensured the resilient operation of the MG against islanding events. In the model, the uncertainties associated with load and intermittent generation resources were captured via a scenario-based stochastic approach. Demand behavior in response to time-varying prices was modeled via price elasticity coefficients. Also, effectiveness of the proposed model was investigated through extensive numerical studies and sensitivity analyses.
\end{abstract}

(C) 2019 Sharif University of Technology. All rights reserved.

\section{Introduction}

Capacity deficit in electric power systems may lead to the failure of almost every critical infrastructure such as food, energy, water, ICT, transport, health, emergency services, government, and finance, to name a few [1]. This is in line with the statement of the National Academy of Sciences of the United States mentioning that the modern world runs on electricity [2]. This pivotal role of electric power systems reflects the necessity for approaches to protecting electric power systems against habitual and occasional events and threats, especially on the evidence of the recent increase

*. Corresponding author. Tel.: +982166165931 E-mail addresses: Mohsen.chegenizadeh@ee.sharif.edu ( $M$. Chegnizadeh); safdarian@sharif.edu (A. Safdarian)

doi: 10.24200 /sci. 2019.54235 .3657 in manmade and natural disasters [3,4]. However, these events occur more frequently as a consequence of climate changing and they are remote from daily experiences with surprising characteristics in areal extent, severity, and time. Needless to mention, such new alien and out of ordinary challenges [2-6] may impose new risks to electric power systems [7]. The risks are imposed mainly because of the low probability and predictability of the events [7]. These characteristics scrap the traditional, clichéd risk-based approaches intended for ensuring that electric power systems are safely operated. With this in mind, engineers were prompted to develop a new resiliency paradigm [7] based on the idea that, although stress could not be clearly anticipated, the associated risks would be reduced by preventive and adaptive goals [8]. This approach, in both technical and institutional respects, was expanded upon the spectrum of events and threats originating from natural disasters, accidents, and de- 
liberate attacks. Nowadays, resiliency is present in the strategic goals of the United States and Canada joint security strategy [8], the strategic plan of the United States Department of Energy [9], and some other technical plans.

To enhance resiliency of the power system, the approaches to dealing with different types of threats are classified into two categories of operation-oriented [10] and hardening-oriented [11]. Some of the approaches only touch reinforcement of elements against a specific type of threat $[12,13]$ while others revolve around solutions that can be implemented independent of the threat [14], one of which is using microgrids (MGs) [15].

MG was initially introduced to facilitate proliferation of local generation, including renewable and conventional resources, offering diverse benefits by meeting local controllable demands in an efficient, reliable, resilient, and environmentally friendly manner $[16,17]$. The conspicuous feature of an MG, called islanding, is its ability to cope with the risky situations where support from the upstream power system is unavailable [18-20].

In order to design an $\mathrm{MG}$, different objectives such as cost minimization, reliability improvement, and environmental concern alleviation can be pursued. Bahramirad et al. [21] proposed a model for sizing the storage while trying to maintain the trade-off between reliability and cost. A model for MG expansion to optimally size the storages with respect to some practical factors was proposed by Alsaidan et al. [22]. It is worthwhile to note that reliability is a very important issue for MGs hosting high penetrations of intermittent and uncertain renewable energy resources [23]. Madathil et al. [24] addressed the N-1 reliability of capacity planning and operations for remote small MGs. Billinton [25] presented a discussion of the impact of renewable energy resources on reliability of the islanded electric power systems. In [26], a model has been proposed to identify the optimum structure of MGs by siting switches in an electric power distribution network. Shahidehpour and Khodaei [27] presented a model to design an $\mathrm{MG}$ by considering the impact of uncertainties associated with renewable energy resources, load, market prices, and islanding. To consider these uncertainties, they optimized the worst-case situation via Benders decomposition technique. In [28], a bi-level robust optimization model is developed in order to optimize participation of MGs in the markets as well as operation of energy resources. To consider uncertainties, a genetic algorithm has been used in this study. In [29] and [30], the focus is on planning MGs with environmental objectives considering incentives and penalties for greenhouse gas emissions.

In [31], a model is presented to design transmission network and MGs, simultaneously, in order to minimize the costs. The study also minimizes load curtailment in the case of faults. Cheng and Wang [32] tried to maximize profit of the $\mathrm{MG}$ owner as the purpose of their design problem. In order to ensure reliability of the designed MG, they considered a lower bound of the percentage of load supplied via local power generation.

In [33], resilient operation of MGs is studied and an $\mathrm{MG}$ is called resilient if its hosting load is served during unexpected islanding events. In order to ensure resiliency of an $\mathrm{MG}$, backup generation units are proposed to be installed to compensate for the intermittent nature of renewable energy resources. Needless to mention, MG owners are not in favour of the installation of the backup units, which significantly increases the costs. To resolve this issue, Khodaei [34] investigated a provisional MG concept by which a few MGs could be operated in connected mode to support each other during islanding events.

Controllable loads play an indispensable role in addressing the coordination between supply and demand in $\mathrm{MG}$, where decision making is intertwined with uncertainties associated with forecasting. In this regard, Atia and Yamada [35] incorporated several controllable loads of the residential MG into sizing of the renewable and energy storage problem. Wu et al. [36] focused on a two-stage robust MG coordination strategy. They scheduled a day-ahead price-based Demand Response (DR) program assuming that micro-turbine outputs were modified on an hourly basis. In [37], a mathematical operational DR model for isolated MG has been offered in which any household owns an intelligent Energy Hub Management System (EHMS) to minimize its costs, while the objective function of the MG operator is overall cost. Ahmad Bashir et al. [38] proposed a novel energy management framework to minimize the total MG cost. For preserving comfort priorities of individual households by a smart central management system, they combined proactive and reactive approaches in order to efficiently address the uncertainties associated with generation and demand in the interconnected operation and random outages of MG components.

The review of studies above emphasizes that MG is a structure with great benefits for all stakeholders in many ways. However, under the islanding mode, hosting a great number of intermittent resources may result in challenging balancing issues [34], which necessitates expensive back-up generators.

In the literature, there is no significant research focusing on methods to avoid the expensive solution of backup generation units. To fill this gap, this paper proposes using demand response potentials to relieve the need for such expensive facilities, especially during island operation mode. This also enhances MG resiliency since critical loads hosted by the MG can be served while the upstream grid is unavailable due 
to manmade or natural disasters. For this purpose, a mathematical model is developed in this paper in which demand response is integrated into MG design process. To avoid computational complexities, the model is decomposed into a master problem and two types of sub-problems via Benders decomposition approach. The master problem makes decisions on binary variables, while the first type of sub-problems optimizes continuous variables and the second type investigates whether MG resilience is maintained within island operation mode. The performance of the model is verified through extensive numerical studies and several sensitivity analyses.

The rest of this paper is organized as follows. Section 2 presents the concept of the model and key assumptions made. Section 3 provides mathematical formulation of the model. Section 4 presents numerical studies and discussions. Finally, relevant conclusions are drawn in Section 5.

\section{Methodology outline}

\subsection{Overview}

The model for incorporating demand response potentials enabled via dynamic prices in $\mathrm{MG}$ planning studies is demonstrated in Figure 1. It aims at maximizing profit of the $\mathrm{MG}$ owner while the operating point is able to ensure that the MG is resilient to the islanding event. The problem contains binary decision variables associated with installation. Moreover, the response of consumers to dynamic prices makes the problem nonlinear. Therefore, the problem in hand is a mixed-integer non-linear one clearly hard to solve. To cover non-linearity of the problem and ensure affordability of the solution to its computational complexity, Benders decomposition method is employed to decompose the problem into a master problem and two types of sub-problems. In the master problem, the investment binary decision variables are determined. The number of sub-problems in the first set is equal to the total number of time intervals. The investment plan decided in the master problem is employed for the time interval sub-problems, called operation subproblems hereinafter, to find the optimal schedule for the operational variables during the time interval. The sub-problems of the second type, called resiliency subproblems hereinafter, check the safe operation of the MG during islanding event. The decision variables are mentioned below:

- Investment variable decisions include the investment states of dispatchable and non-dispatchable distributed generation as well as energy storage units. These decisions are modelled via binary variables, which are optimized by minimizing the total investment cost. Note that the binary variables are set to one for installation, zero otherwise. In addition, it is worthwhile to mention that generation and storage units are chosen from the set of introduced candidates;

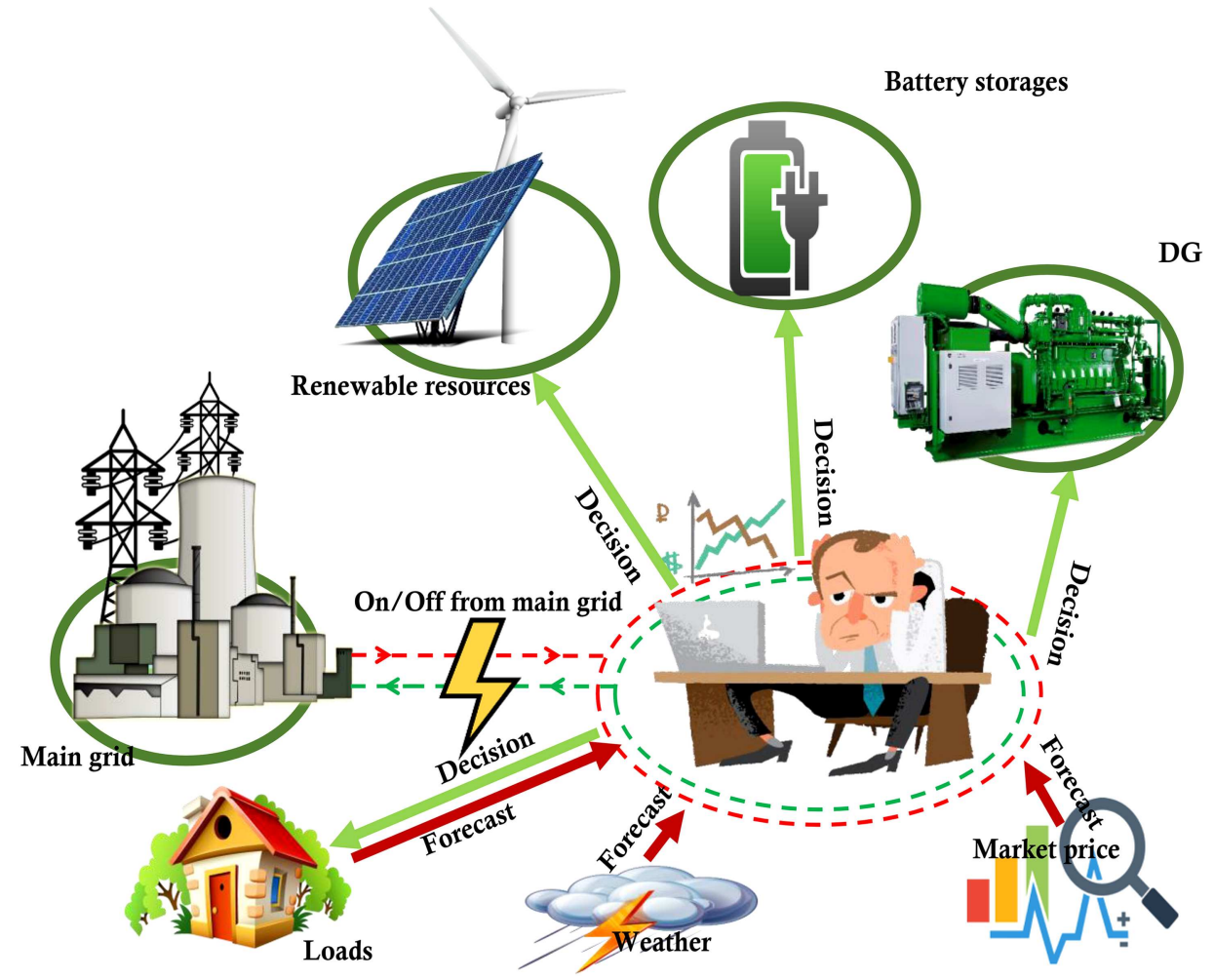

Figure 1. Diagram of the proposed MG decision making model. 
- Operation variable decisions comprise the active power output of dispatchable units, the amount of charge/discharge power of storage units, the amount of load, and power transaction between the MG and the upstream grid. It is clear that these decisions are made in line with the maximization of the profit of the MG owner.

In the model, the uncertainty associated with load and intermittent generation resources is considered via a scenario-based stochastic approach. The demand behaviour in response to price changes is captured via price elasticity coefficients.

Figure 2 depicts the flowchart of the proposed MG planning model. As can be seen, the problem is solved via an iterative approach by which the master problem and sub-problems are solved sequentially. The solution to the master problem is fed to the sub-problems and the results achieved by solving the sub-problems are used to revise the master problem. The solution convergence is examined based on the closeness of a calculated investment cost in the master problem and the profit that is calculated in the operation subproblems. The iterative process continues until the convergence criterion is met. Finally, the investment and operation variables are assessed in the resiliency

\section{Determine optimal DER} generation

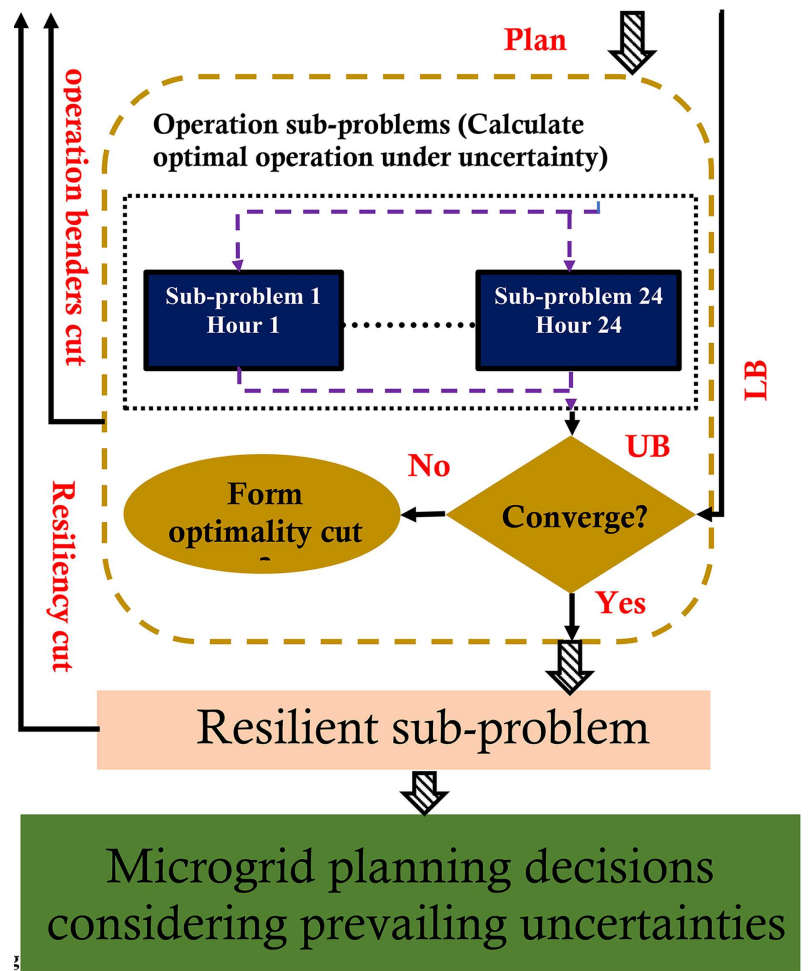

Figure 2. Flowchart of the proposed MG planning model. sub-problem to ensure generation adequacy for the islanding event. If the generation is not adequate, the master problem is revised so as to ensure the necessary condition for the islanding event.

\subsection{Assumptions}

The principal assumptions of the proposed model are mentioned as follows.

- The MG operator is the owner of all installed assets and facilities. Synonymously, the $\mathrm{MG}$ owner and operator are the same in this study;

- Ramp limitations and costs are not considered for dispatchable units in this study. This makes sense because of the small size and rapid responding capability of such units;

- Dynamic prices are employed to coordinate supplies and demands. The demand behaviour in response to price changes is captured via price self- and crosselasticity coefficients, which are considered to be constant parameters in the planning horizon;

- Since the MG operator and the owner are the same, the generation side of the $\mathrm{MG}$ is not an open market. Hence, it introduces regulatory constraints on hourly prices and yearly income to preserve fairness;

- Costs of system maintenance and smart meter installations are ignored. In other words, it is assumed that the metering infrastructures are already installed in the system;

- No salvage value is considered since useful life of facilities is supposed to be similar to the planning horizon;

- As local generation is close to consumption, network congestion is ignored and the system is supposed to be a balanced three-phase system. These simplifications are to underline the major contribution of this paper;

- The Point of Common Coupling (PCC), which connects the $\mathrm{MG}$ to the main grid, is assumed to have enough capacity to supply the MG load during the entire planning horizon;

- In order to ensure the possibility of the contribution of energy storages in the islanded mode, it is assumed that MG operator keeps the energy stored in batteries at the scheduled plan. This assumption is for avoiding the effect of uncertainties associated with renewable resources on the energy stored in batteries.

\section{Mathematical formulation}

In this section, mathematical formulation of the proposed original model is first presented. Then, the 
solving procedure is elaborated on, through which the original problem is decomposed into the master problem and operation and resiliency sub-problems.

\subsection{Original model}

As mentioned earlier, the objective of the model is to maximize total profit of the MG owner through the planning horizon as follows:

$$
\text { MAX } R-\cos t \text {. }
$$

In Relation (1), the profit is represented as the revenue item minus costs. Revenue is the outcome of selling energy to consumers while the cost function consists of annual capital costs, operation costs, and costs of the unserved energy. The maximum profit is evaluated in terms of discounted values by the incorporation of the present-worth costs or revenue components. Revenue and cost are formulated as follows:

$$
\begin{aligned}
& \cos t=\sum_{y} \sum_{i \in(G, W)} k_{y} C C_{i y} P_{i}^{\max } x_{i} \\
& +\sum_{y} \sum_{i \in S} k_{y}\left(C P_{i y} P_{i}^{\max }+C E_{i y} C_{i}^{\max }\right) x_{i} \\
& +\sum_{y} \sum_{d} \sum_{h} \sum_{s \in S} \sum_{i \in G} k_{y} \omega_{s}\left(c_{i} p_{i y d h s}\right) \\
& +\sum_{y} \sum_{d} \sum_{h} \sum_{s \in S} k_{y} \omega_{s}\left(\rho_{y d h s} p_{M, y d h s}\right) \\
& +\sum_{s \in S} \sum_{y} \sum_{d} \sum_{h} k_{y} v_{y d h} L S_{y d h s} \\
& R=[P]_{1 \times 24}^{t} \times[\lambda]_{24 \times 1}, \\
& P_{h}=P_{h}^{\text {flat }}\left[1+\sum_{h^{\prime} \in H} E_{h, h^{\prime}}^{t} \frac{\lambda_{h^{\prime}}-\lambda^{\text {flat }}}{\lambda^{\text {flat }}}\right], \\
& P_{h}=P_{h}^{\text {flat }}\left[1+e_{h, 1} \frac{\lambda_{1}-\lambda^{\text {flat }}}{\lambda^{\text {flat }}}+e_{h, 2} \frac{\lambda_{2}-\lambda^{\text {flat }}}{\lambda^{\text {flat }}}\right. \\
& +\ldots+e_{h, h} \frac{\lambda_{h}-\lambda^{\text {flat }}}{\lambda^{\text {flat }}}+\ldots \\
& \left.+e_{h, 24} \frac{\lambda_{24}-\lambda^{\text {flat }}}{\lambda^{\text {flat }}}\right] \\
& R_{h}=\lambda_{h} \times P_{h}^{\text {flat }}\left[1+e_{h, 1} \frac{\lambda_{1}-\lambda^{\text {flat }}}{\lambda^{\text {flat }}}\right. \\
& +e_{h, 2} \frac{\lambda_{2}-\lambda^{\text {flat }}}{\lambda^{\text {flat }}}+\ldots+e_{h, h} \frac{\lambda_{h}-\lambda^{\text {flat }}}{\lambda^{\text {flat }}}+\ldots \\
& \left.+e_{h, 24} \frac{\lambda_{24}-\lambda^{\text {flat }}}{\lambda^{\text {flat }}}\right],
\end{aligned}
$$

$$
\begin{aligned}
\frac{R_{h}}{P_{h}^{\text {flat }}}= & \lambda_{h}+\left(\frac{e_{h, 1}}{\lambda^{\text {flat }}} \times \lambda_{h} \times \lambda_{1}+\frac{e_{h, 2}}{\lambda^{\text {flat }}} \times \lambda_{h} \times \lambda_{2}\right. \\
& \left.+\ldots+\frac{e_{h, h}}{\lambda^{\text {flat }}} \times \lambda_{h}^{2}+\ldots+\frac{e_{h, 24}}{\lambda^{\text {flat }}} \times \lambda_{h} \times \lambda_{24}\right) \\
& -\left(e_{h, 1}+e_{h, 2}+\ldots+e_{h, h}+\ldots+e_{h, 24}\right) \times \lambda_{h},
\end{aligned}
$$

$$
\begin{array}{rl}
R_{T}= & \sum_{h \in H} \frac{R_{h}}{P_{h}^{\text {flat }}}, \\
R_{T}= & \left\{\sum_{h \in H} \sum_{\substack{h^{\prime} \in H \\
\forall h^{\prime} \neq h}} P_{h}^{\text {flat }} \times \frac{e_{h, h^{\prime}}}{\lambda^{\text {flat }}} \times \lambda_{h} \times \lambda_{h^{\prime}}\right\} \\
& +\left\{\sum_{h \in H}\left(\lambda_{h} \times P_{h}^{\text {flat }} \times\left\{1-\sum_{h^{\prime} \in H} e_{h, h^{\prime}}\right\}\right)\right\} \\
& +\left\{\sum_{h \in H} P_{h}^{\text {flat }} \times \frac{e_{h, h}}{\lambda^{\text {flat }}} \times \lambda_{h}^{2}\right\} \\
e_{i, j}= & e_{j, i}, \quad i \in P_{i}, \\
\alpha a_{i}= & P_{i}^{\text {flat }} \times \frac{e_{i, i}}{\lambda^{\text {flat }}} ; \quad i \in H \\
\beta a_{i, j}= & \frac{\left(P_{i}^{\text {flat }}+P_{j}^{\text {flat }}\right) \times e_{i, j}}{\lambda^{\text {flat }}} ; \\
i \in P_{i} & H, j \in H, i \neq j \\
v_{i}=\sum_{h^{\prime} \in H}^{\text {flat }}\left(1-e_{i, h^{\prime}}\right) ; \quad i \in
\end{array}
$$

The operation cost presented in Eq. (2) is composed of cost of the selected non-renewable generation and the costs of power imports from the main grid. The revenue is calculated through multiplying selling price by the demand of costumers during time (Eq. (3)). Because the demand and price are interdependent, employing demand response program makes these items intertwined. As a result, changing price leads to demand shift from/to the relevant hour. This makes the model nonlinear. In this regard, the demand and price formulations are extended, and nonlinear items in the revenue formula are detected and linearized. The extended form of the demand is given in Eqs. (4) and (5). The demand at time interval $h$ is computed by the elasticity matrix. The elasticity matrix includes self and cross elasticity coefficients that represent capability of the demand to shift from/to other time intervals based on price changes. This matrix is applied to the initial demand in Eq. (4) and in detail in Eq. (5). Substituting Eq. (5) in Eq. (3), the revenue can be achieved by Eqs. (6) and (7). In addition, total revenue, 
represented in Eq. (8) and summarized in a compact form in Eq. (9), can be formulated as follows by using the auxiliary parameters defined in Eqs. (10)-(13):

$$
\begin{aligned}
R_{T}= & \left\{\sum_{h \in H} \sum_{\substack{h^{\prime} \in H \\
\forall h^{\prime} \neq h}} \beta a_{h, h^{\prime}} \times \lambda_{h} \times \lambda_{h^{\prime}}\right\} \\
& +\left\{\sum_{h \in H}\left(\lambda_{h} \times v_{h}\right)\right\}+\left\{\sum_{h \in H} \alpha a_{h} \times \lambda_{h}^{2}\right\} .
\end{aligned}
$$

Needless to mention, coping with the nonlinearity of the first and third items of Eq. (14) plays a crucial role in the computational feasibility. As a result, the technique proposed in [39] is applied to the first term in Eq. (14) as follows:

$$
\beta a_{i, j} \times \lambda_{i} \times \lambda_{j}=\frac{\beta a_{i, j}}{2} \times\left\{\left(\lambda_{i}+\lambda_{j}\right)^{2}-\lambda_{i}^{2}-\lambda_{j}^{2}\right\} .
$$

The linearization is followed by defining $\theta_{h}$ as follows and sorting revenue expression in Eq. (17):

$$
\begin{aligned}
\theta_{m}= & \alpha a_{m}-\sum_{h^{\prime} \in H} \frac{\beta a_{m, h^{\prime}}}{2}, \\
R_{T}= & \left\{\sum_{\substack { h \in H \\
\begin{subarray}{c}{h^{\prime} \in H \\
\forall h^{\prime} \neq h{ h \in H \\
\begin{subarray} { c } { h ^ { \prime } \in H \\
\forall h ^ { \prime } \neq h } }\end{subarray}} \frac{\beta a_{h, h^{\prime}}}{2} \times\left(\lambda_{h}+\lambda_{h^{\prime}}\right)^{2}\right\} \\
& +\left\{\sum_{h \in H}\left(\lambda_{h} \times v_{h}\right)\right\}+\left\{\sum_{h \in H} \theta_{h} \times \lambda_{h}^{2}\right\} .
\end{aligned}
$$

The square items in the first and last terms of Eq. (17) are still non-linear. Therefore, piecewise linear approximation of these terms, as in [39], is used as follows:

$$
\begin{aligned}
& \theta_{h} \lambda_{h}^{2}=\phi_{h, 1} \eta_{h, 1}+\phi_{h, 2} \eta_{h, 2}+\phi_{h, 3} \eta_{h, 3}+\phi_{h, 4} \eta_{h, 4} \\
& \quad h \in H, \\
& \lambda_{h}=\eta_{h, 1}+\eta_{h, 2}+\eta_{h, 3}+\eta_{h, 4}, \\
& \phi_{h, l}=2 \times \theta_{h} \lambda_{h}, \\
& \frac{\beta a_{h, h^{\prime}}}{2} \times\left(\lambda_{h}+\lambda_{h^{\prime}}\right)^{2}=\varphi_{1, h, h^{\prime}} \chi_{1, h, h^{\prime}}+\varphi_{2, h, h^{\prime}} \chi_{2, h, h^{\prime}} \\
& \quad+\varphi_{3, h, h^{\prime}} \chi_{3, h, h^{\prime}}+\varphi_{4, h, h^{\prime}} \chi_{4, h, h^{\prime}}, \\
& \lambda_{h}+\lambda_{h^{\prime}}=\chi_{1, h, h^{\prime}}+\chi_{2, h, h^{\prime}}+\chi_{3, h, h^{\prime}}+\chi_{4, h, h^{\prime}}, \\
& \beta a_{h, h^{\prime}} \times\left(\lambda_{h}+\lambda_{h^{\prime}}\right)=\varphi_{y, h, h^{\prime}} ; \quad y=1,22,3,4,
\end{aligned}
$$

where the non-linear problem is divided into 4 linear sections with slopes $\varphi_{h, l}$ and $\varphi_{y, h, h^{\prime}}$. Such linearized problem should be solved along with several operational and investment limitations. In order to ensure that such restrictions be materialized, constraints (24)(39) are applied to the linearized problem as follows:

$$
0 \leq p_{i h} \leq p_{i}^{\max } \widehat{x}_{i} ; \quad \forall h, \forall i \in G
$$

Constraint (24) is to consider the minimum and maximum generation capacity limits of installed dispatchable units. In addition, the following constraints are considered to formulate the limits on a battery storage:

$$
\begin{aligned}
& 0 \leq p_{i, h}^{c h} \leq p_{\max }^{c h} U_{i, t} ; \quad \forall h, \forall i \in E \\
& 0 \leq p_{i, h}^{d c h} \leq p_{\max }^{d c h} V_{i y d h} ; \quad \forall h, \forall d, \forall y, \forall i \in E \\
& U_{i y d h}+V_{i y d h} \leq 1 ; \quad \forall y, \forall d, \forall h, \forall i \in E \\
& 0 \leq C_{i h} \leq C_{i}^{\max } \widehat{x}_{i} ; \quad \forall h, \forall i \in E \\
& C_{i, t}=C_{i, h-1}+\eta p_{i, h}^{c h}-(1 / \eta) p_{i, h}^{d c h} ; \quad \forall h, \forall i \in E \\
& 0 \leq V_{i y d h} \leq \widehat{x}_{i} ; \quad \forall h, \forall d, \forall y, \forall i \in E \\
& 0 \leq U_{i y d h} \leq \widehat{x}_{i} ; \quad \forall h, \forall d, \forall y, \forall i \in E
\end{aligned}
$$

The operational limits of storage units are described in Constraints (25)-(31). Constraints (25) and (26) are to incorporate charge/discharge limits of storage unit while Constraint (27) ensures that the units are not charged and discharged simultaneously. The state of charge of the storage units is calculated in Constraint (28). Constraint (29) is to ensure that the energy saved in the units is within the upper and lower limits. Constraints (30) and (31) guarantee that only the storage units chosen to be installed make contribution to the system operation. Besides the aforementioned constraints, the problem is faced with some technical restrictions given below:

$$
\begin{aligned}
&-p_{M}^{\max } \leq p_{M h} \leq p_{M}^{\max } ; \quad \forall h \\
& \sum_{i \in(G, W)} P_{i y d h s}+\sum_{i \in S}\left(P_{i y d h s}^{d c h}-P_{i y d h s}^{c h}\right)+P_{M, y d h s} \\
&+L S_{y d h s}=D_{y d h s}, \\
& \forall h, \forall d, \forall y, \forall k, \forall s \in S .
\end{aligned}
$$

Constraint (32) ensures that the power exchanged with the main grid is limited by the associated limit at PCC. Equation (33) enforces maintaining a balance between generation and consumption in each time period. 
Similarly, revenue and profit must be restricted by under-regulation constraints so as to preserve fairness as follows:

$$
\begin{aligned}
& \lambda_{y k t \omega}=\lambda_{y k t \omega}^{\text {service }}+\lambda_{y k t \omega}^{\text {energy }}, \\
& \lambda_{y k t \omega}^{\text {service }} \leq \lambda_{k}^{\text {service }}, \\
& \sum_{y} \sum_{k} \sum_{t} \lambda_{y k t \omega}^{\text {service }} P_{i y k t \omega}^{D} \leq \lambda^{\text {average }} \\
& \quad \times \sum_{y} \sum_{k} \sum_{t} P_{i y k t \omega}^{D} .
\end{aligned}
$$

Constrains (34)-(36) give regulatory constraints on selling prices and yearly income of the MG owner. For this purpose, price in any time interval is divided into service and energy parts. The energy price is equal to the market price in the same time interval, while the MG owner determines the service price. Constraint (35) puts a cap over the service price.

Now, the proposed solving procedure is introduced in the next sub-section, where the original linearized model is decomposed into a master problem and operation and resilient sub-problems by Benders decomposition technique.

\subsection{Solving procedure}

\subsubsection{Master problem}

The objective function of the master problem is composed of the first two items in Eq. (2). This problem is solved on a yearly basis aiming at minimizing the investment costs (Constraint (37)). Thereby, decision variables related to the installation state of distributed generation and storage units are given values in this problem. The results are then sent to the operation sub-problems. In Constraint (37), variable $\Lambda$ is a projected operation cost in the investment problem. This sub-problem calculates the upper-bound of the Benders decomposition problem:

$$
\begin{aligned}
\operatorname{Min} \sum_{y} & \sum_{i \in(G, W)} K_{y} C C_{i y} P_{i}^{\max } x_{i} \\
& +\sum_{y} \sum_{i \in S} K_{y}\left(C P_{i y} P_{i}^{\max }+C E_{i y} C_{i}^{\max }\right) x_{i} \\
& +\sum_{y} k_{y} \Lambda_{y} .
\end{aligned}
$$

\subsubsection{Operation sub-problems}

In computing the lower bound, the operation subproblems are in charge of scheduling selling prices, charge/discharge power of storage units, output power of dispatchable generation units, and power imports from the main grid with the aim of maximizing profit of the MG owner. Accordingly, the objective function of the operation sub-problem comprises the third and fourth items in Eq. (2) with respect to the master- level decisions and in compliance with the following constraints:

- Generation capacity constraints (Constraint (24));

- Power and energy limitations of storage units (Constraints (25)-(31));

- System power balance and PCC limitation (Constraints (32)-(33));

- Regulatory constraints (Constraints (34)-(36)).

Having compared lower and upper bounds, if the convergence criterion is not met, the feasible operation cut is formed as follows:

$$
\begin{aligned}
\Lambda_{y} & \geq \widehat{Q}_{y}+\sum_{d} \sum_{h} \sum_{i \in G} \delta_{i y d h} P_{i}^{\max }\left(x_{i, t}-\widehat{x}_{i, t}\right) \\
& +\sum_{d} \sum_{h} \sum_{i \in W} \varsigma_{i y d h} P_{i}^{\max }\left(x_{i, t}-\widehat{x}_{i, t}\right) \\
& +\sum_{d} \sum_{h} \sum_{i \in S} \vartheta_{i y d h} P_{i}^{\max }\left(x_{i, t}-\widehat{x}_{i, t}\right) \\
& +\sum_{d} \sum_{h} \sum_{i \in G} \tau_{i y d h} C_{i}^{\max }\left(x_{i, t}-\widehat{x}_{i, t}\right)
\end{aligned}
$$

where $\widehat{Q}_{y}$ is a calculated objective value of the operation sub-problems in a year and $\delta_{i y d h}, \zeta_{i y d h}, \vartheta_{i y d h}$, and $\tau_{i y d h}$ are dual variables associated with dispatchable generation units, non-dispatchable generation units, rated power of storage units, and rated capacity of storage units. Added to the master problem in the next iteration, the cut revises the combination of installing units.

\subsubsection{Resiliency sub-problem}

Calculated in the master problem and operation subproblems, the optimal investment plan and scheduled operation are assessed in the resiliency sub-problem to ensure energy adequacy for the islanding event. By doing so, the MG is capable of supporting critical loads for $t_{\text {island }}$ hours as the expected continuous time during which the MG is not supported by the main grid. The resiliency sub-problem formulation is as follows:

$$
\begin{gathered}
\operatorname{Min} w=\sum_{t} S L_{1, y d h}+S L_{2, y d h}, \\
\sum_{h=t_{\text {start }}} \sum_{i \in G} P_{i y d h s}+\sum_{i \in S}\left(P_{\text {iydd }_{\text {start }} s}^{d c h}-P_{\text {iyd }_{\text {start }} s}^{\text {ch }}\right) \\
t_{\text {island }}+S L_{1, y d t_{\text {start }}}-S L_{2, y d t_{\text {start }}} \\
\geq \sum_{h=t_{\text {start }}}^{t_{\text {start }}+t_{\text {stand }}} D_{y d h}^{\text {critical }}
\end{gathered}
$$

$\forall h, \forall d, \forall y$, 


$$
\widehat{x}=x_{i} \quad \forall i \in E S, G
$$

where $S L s$ are positive slack variables, $D^{\text {critical }}$ refers to the critical load of the planned $\mathrm{MG}$, and $t_{\text {start }}$ is the time when islanding happens. Eq. (39) minimizes the mismatches that occur in Relation (40). Here, Relation (40) is to ensure that the net energy provided by dispatchable units and charge/discharge of battery storage units is sufficient to support critical load hosted by the MG during the islanding period. The sub-problem must also meet the following constraints according to the master-level decisions (Eq. (41)):

- Power and energy limitations of storage units (Constraints (25)-(31));

- System power balance (Constraint (32)).

Having the sub-problem solved, the final solution is obtained if the mismatch is zero. Otherwise, the solution must be revised. The revision is performed via changing the investment plan by forming resiliency cut (Relation (42)) and sending it back to the master problem.

$$
\begin{aligned}
\widehat{w} & +\sum_{t} \sum_{i \in G} \pi_{i, t}\left(x_{i, t}-\widehat{x}_{i, t}\right) \\
& +\sum_{t} \sum_{i \in S} \mu_{i, t}^{c h}\left(x_{i, t}-\widehat{x}_{i, t}\right) \\
& +\sum_{t} \sum_{i \in S} \mu_{i, t}^{d c h}\left(x_{i, t}-\widehat{x}_{i, t}\right)<0,
\end{aligned}
$$

where $\pi$ and $\mu_{i, t}$ are dual variables associated with binary variables of dispatchable generation units and rated power of storage units, respectively.

\section{Numerical results}

In this section, the proposed methodology is applied to a test system. In order to demonstrate the framework of the simulated $\mathrm{MG}$, the corresponding diagram of the proposed model is depicted in Figure 1. An MG is to be installed for a group of electricity consumers with a peak annual load of 8.5 MW. The set of candidates includes 11 DERs (consisting of 6 dispatchable units, 2 non-dispatchable units, and 3 energy storage systems). The characteristic data of energy storage units are provided in Table 1. Table 2 presents deterministic parameters of the dispatchable units. Similarly, the technical data associated with non-dispatchable units are given in Table 3, illustrating qualities of wind and solar generators.

Energy storage efficiency is considered to be $90 \%$ for all candidates. The load, variable renewable generation, and market prices are forecasted based on the historical data obtained from IIT Campus Microgrid [34]. The planning horizon is 20 years.

The flexible and critical loads are considered 40 and $60 \%$, respectively. In order to avoid computational complexities, any year, which is 8760 hours, is divided up into 8 periods. The reason behind choosing 8 periods is that all workdays in a week during one

Table 1. Characteristics of the energy storage units.

\begin{tabular}{ccccc}
\hline Unit no. & $\begin{array}{c}\text { Rated power } \\
(\mathbf{M W})\end{array}$ & $\begin{array}{c}\text { Rated energy } \\
(\mathbf{M W h})\end{array}$ & $\begin{array}{c}\text { Annualized investment } \\
\text { cost-power }(\mathbf{\$} / \mathbf{M W})\end{array}$ & $\begin{array}{c}\text { Annualized investment } \\
\text { cost-energy }(\$ \mathbf{M W h})\end{array}$ \\
\hline 1 & 1 & 6 & 60000 & 30000 \\
2 & 2 & 6 & 30000 & 30000 \\
3 & 3 & 6 & 20000 & 30000 \\
\hline
\end{tabular}

Table 2. Characteristics of dispatchable units.

\begin{tabular}{cccc}
\hline Unit no. & $\begin{array}{c}\text { Rated power } \\
(\mathbf{M W})\end{array}$ & $\begin{array}{c}\text { Cost coefficient } \\
(\$ / \mathbf{M W})\end{array}$ & $\begin{array}{c}\text { Annualized investment } \\
\text { cost }(\$ \mathbf{M W h})\end{array}$ \\
\hline 1 & 5 & 90 & 50000 \\
2 & 5 & 90 & 50000 \\
3 & 3 & 70 & 70000 \\
4 & 3 & 70 & 70000 \\
5 & 2 & 60 & 100000 \\
6 & 2 & 60 & 100000 \\
\hline
\end{tabular}

Table 3. Characteristics of non-dispatchable units.

\begin{tabular}{cccc}
\hline Unit no. & $\begin{array}{c}\text { Rated power } \\
(\mathbf{M W})\end{array}$ & $\begin{array}{c}\text { Cost coefficient } \\
(\$ / \mathbf{M W})\end{array}$ & $\begin{array}{c}\text { Annualized investment } \\
\text { cost }(\$ / \mathbf{M W h})\end{array}$ \\
\hline 1 & 2 & 0 & 120000 \\
2 & 2 & 0 & 180000 \\
\hline
\end{tabular}


season experience approximately similar market prices, loads, and renewable generation. Similarly, weekends have such a feature. In this way, the market price, load, and wind and solar generation in one typical day are employed for all days in the related period. Therefore, the problem for every year is solved for 8 days rather than 365 days. Based on the forecasts, the likely wind and solar generation scenarios for spring are illustrated in Figures 3 and 4, respectively. In addition, Figures 5 and 6 demonstrate one scenario of market prices and solar generation in all seasons of the first year, respectively.

As noted earlier, the developed model encourages consumers to reduce their load when the market prices are high or interior renewable generation drains away. As demonstrated in Eq. (4), calculated sale prices in comparison with the relevant flat prices determine how much load should contribute to the demand response program. For this reason, flat prices are defined by

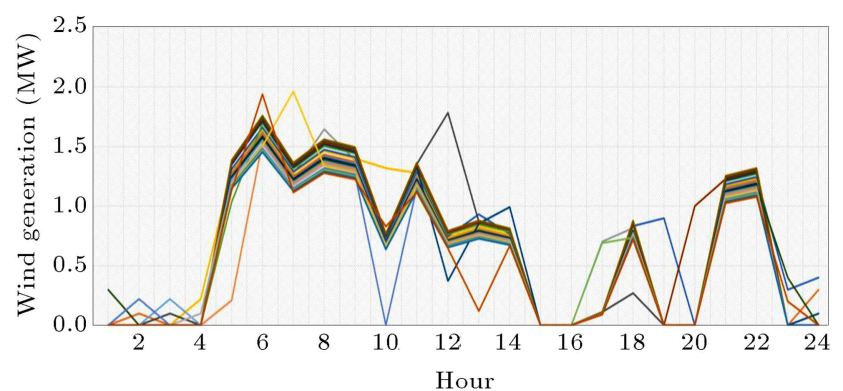

Figure 3. Output of wind turbine generators-spring, first year.

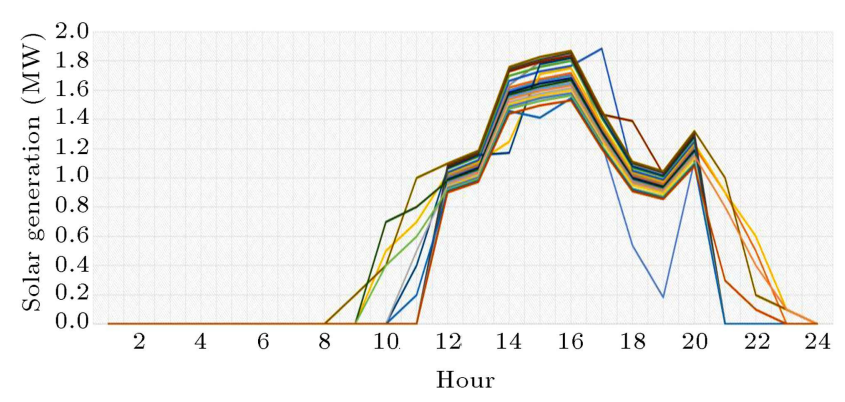

Figure 4. Output power of solar panels-spring, first year.
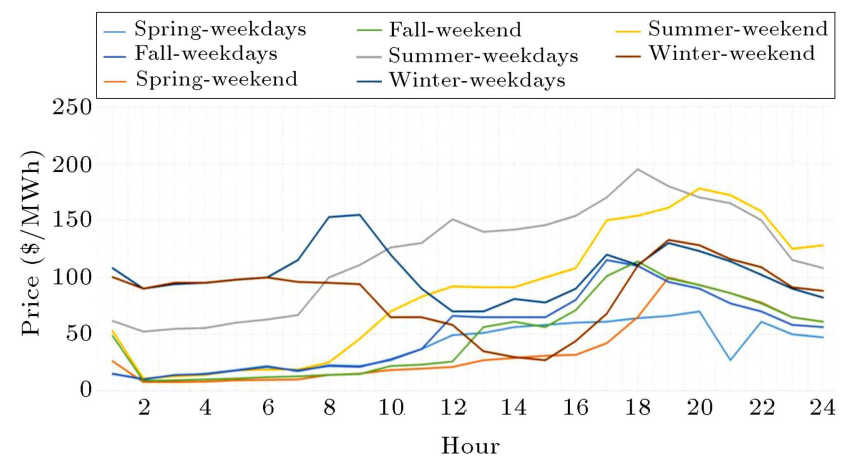

Figure 5. Hourly prices for one scenario-first year.

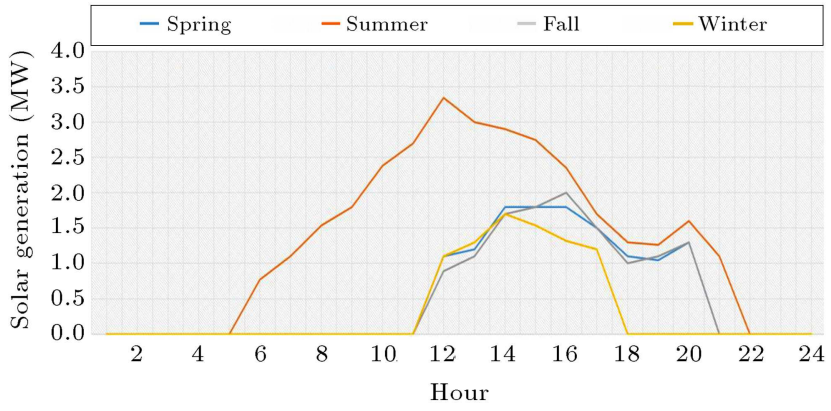

Figure 6. Output power of solar panels-all seasons.

which consumers pay a fixed price for the amount of electricity they consume. To quantify such prices, 2 flat prices are calculated for the peak and off-peak durations of the year (i.e., summer and winter as peak durations, and spring and fall off-peak). Specifically, lower sale prices than flat prices enforce demand to shift to the relevant hours, while higher price shifts demand to other hours. Having calculated the amount of the load shifted at different load points and elasticities, the flat prices are calculated using the following formula:

$$
\lambda^{\text {flat }}=\frac{\sum_{s} \sum_{y} \sum_{d} \sum_{h} \omega_{s} \rho_{s y d h}^{s p o t} P_{s y d h}^{f l l a t}}{\sum_{s} \sum_{y} \sum_{d} \sum_{h} \omega_{s} P_{s y d h}^{f l l a t}},
$$

where $P_{\text {sydh }}^{\text {fllat }}$ and $\rho_{\text {sydh }}^{\text {spot }}$ are base load (without implementing time-varying demand response program) and electricity market price, respectively.

To investigate validity of the method, two cases are considered in the simulations. In the first case, called base case hereinafter, demand response is not enabled. According to the simulations results for the first case, it is economically beneficial to install all candidate DERs, since the installing costs are divided up between several years of the planning horizon and the lack of demand response potentials forces installing dispatchable units to preserve resiliency.

To enable demand response in the second case, the self-elasticity and cross-elasticity coefficients are set to -0.2 and 0.0087 , respectively. This case is simulated. According to the results, dispatchable units 5 and 6 are not installed if demand response potentials are activated. It can also be seen in Table 4 that the operation cost and purchasing cost in the base case are more than the corresponding costs of the case in which demand response is enabled. Needless to say, demand response being disable in the base case, the problem forces the $\mathrm{MG}$ owner to install more units and hence, the operation and investment costs increase. In addition, cost of purchasing power from the main grid increases, because it is impossible to move the loads to lower-price hours. Furthermore, profit decreases since the operation, investment, and cost of purchasing power from the main grid increase. 
Table 4. Comparison between cases studied.

\begin{tabular}{cccc}
\hline Scenario & Profit & Operation cost & $\begin{array}{c}\text { Cost of purchasing power } \\
\text { from the main grid }\end{array}$ \\
\hline $\begin{array}{c}\text { Base case } \\
\text { The case with time-varying } \\
\text { demand response }\end{array}$ & 20275181 & 1628394 & 548871 \\
\hline
\end{tabular}

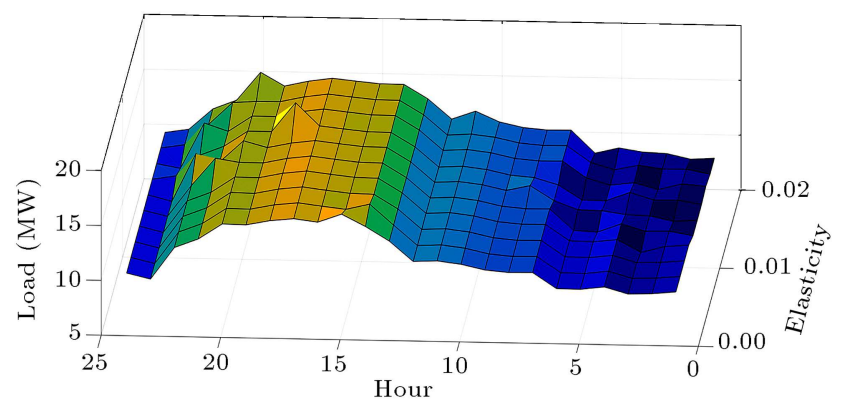

(a)

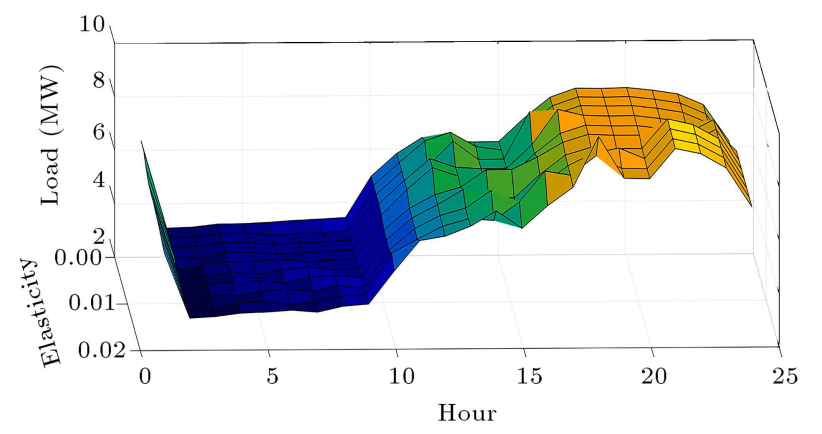

(b)

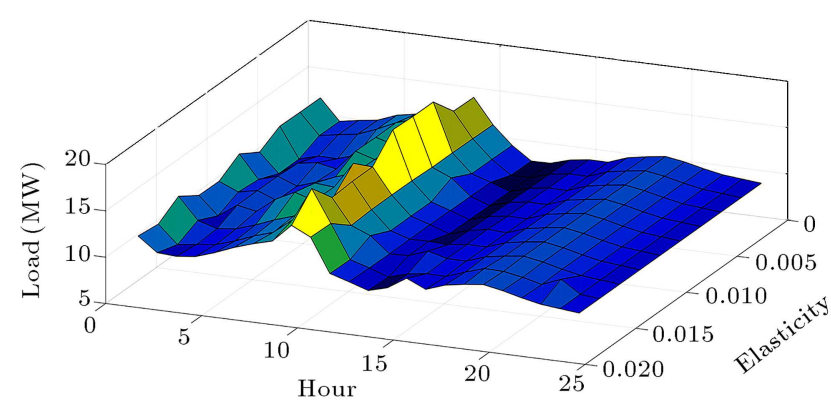

(c)

Figure 7. Simulation results for diverse cross-elasticities: (a) first year, summer, workdays (b) first year, fall, weekend and (c) first year, winter, workdays.

Since the elasticity coefficients are intertwined with hard-to-predict social and economic aspects of human life with time, the simulation is repeated for a wide spectrum of self and cross elasticity coefficients. The obtained load profiles associated with diverse elasticity coefficients are shown in Figure 7(a)-(c). The figures are respectively associated with the simulation results of summer workdays, fall weekends, and winter workdays in the first year of the studied horizon. It

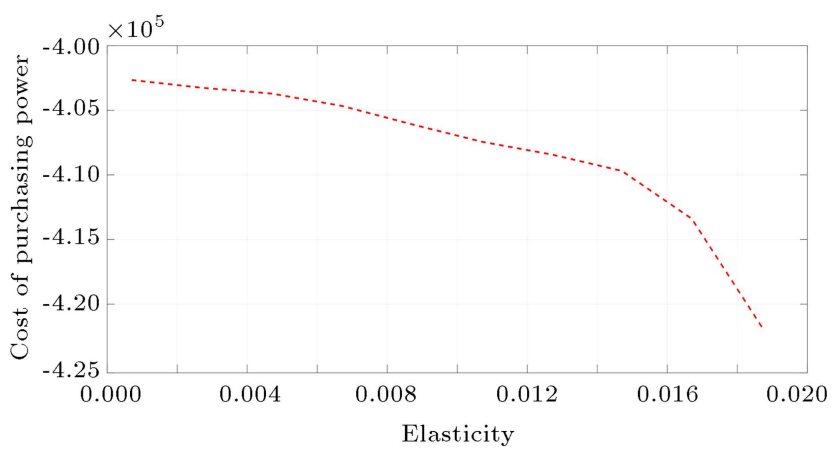

Figure 8. Cost of purchasing power from the main grid for diverse cross-elasticities.

can also be seen in Figure 8 that energy procurement cost from the main grid decreases (negative cost means attaining revenue) by increasing the value of elasticity coefficients. As the maximum revenue that can be earned by the MG owner is fixed by the regulatory constraints, increase in the attained profit translates into decrease in overall costs. This observation reveals the importance of the approach. In Figure $7(\mathrm{a})$, the MG owner is confronted with higher peak demands within the period from $6 \mathrm{pm}$ to $10 \mathrm{pm}$. In case of higher elasticity coefficients (i.e., |cross-elasticity $\mid>0.01$ ), which means that more flexible load is available, this observation is proven. The main reason is that market prices decrease in these hours, while a great number of renewable resources (either solar or wind) are available in the MG. As a result, the proposed problem designs the sale prices in a way to shift demand to these hours. The same results can be seen for hours 21-23 in Figure 7(b) as well as for hours 8-10 in Figure 7(c).

Besides the uncertainty associated with elasticity coefficients, the share of responsive load, which is affected by time-varying prices, is uncertain. The simulation results associated with different shares for responsive load are demonstrated in Figure 9(a)-(c). According to the results, demand response, as a load shaping tool, presents an alternative for the installed generation capacity. As can be seen, the capacity of necessary dispatchable units decreases as the share of responsive load grows. Furthermore, Figure 10 presents the cost of purchasing power from the main grid. It is observable that the cost decreases dramatically as the share of responsive load increases.

Figure 11 shows simulation results for diverse self- 


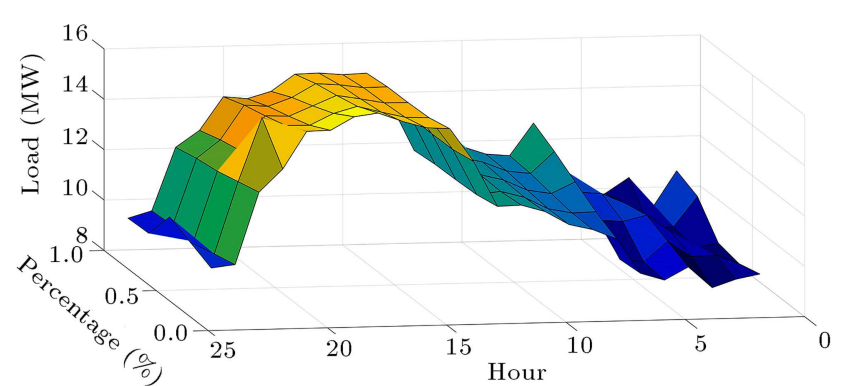

(a)

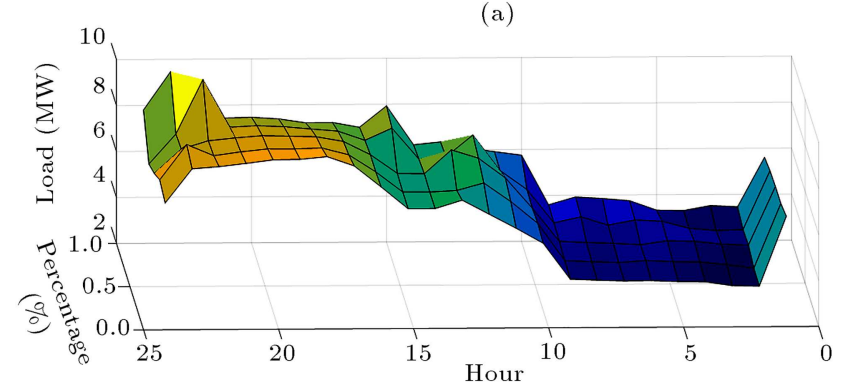

(b)

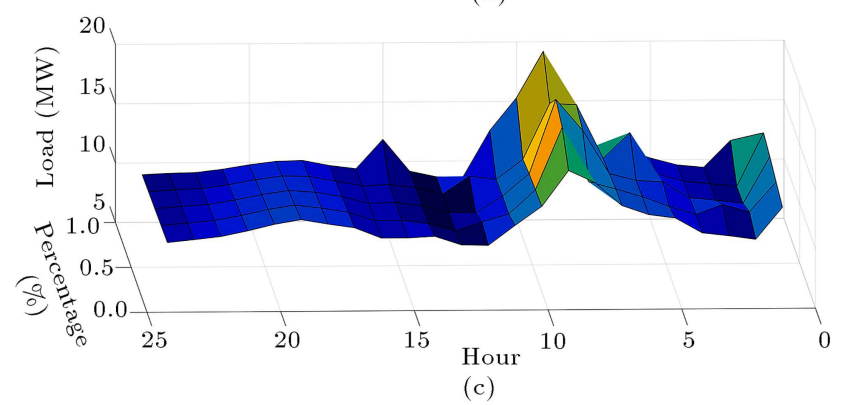

Figure 9. Simulation results for diverse percentages of flexible load: (a) First year, summer, workdays (b) first year, fall, weekend and (c) first year, winter, workdays.

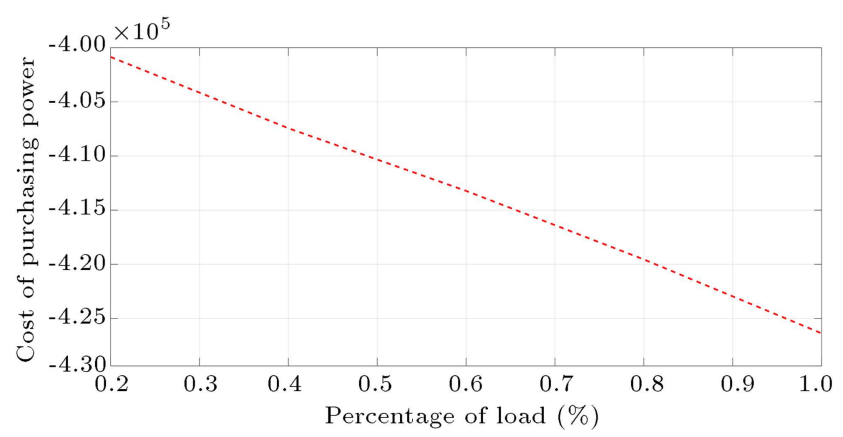

Figure 10. Cost of purchasing power from the main grid for diverse percentages of flexible loads.

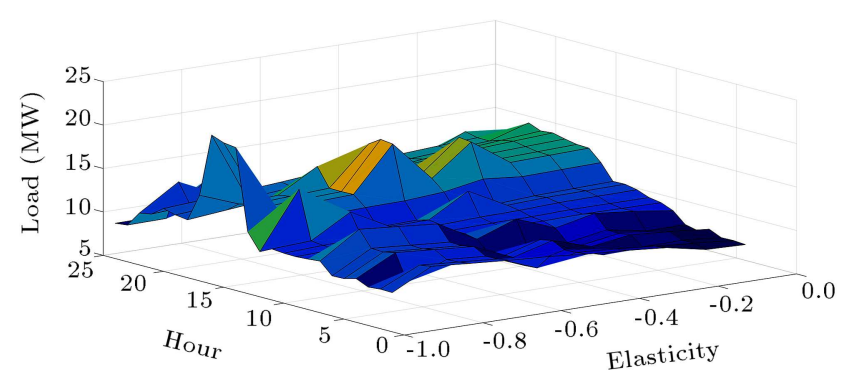

Figure 11. Simulation results for diverse cross-elasticities. elasticities. With reference to this figure, dramatic fluctuations can be seen for hours around 15. The reason can be traced back to Eq. (4), according to which when the sale price is near $\lambda^{\text {flat}}$, minor changes in price lead to change in the second part. As a result, considerable increase or decrease in load can be observed, especially when the problem is faced with higher elasticity coefficients. Captured via price elasticity coefficients, demand behavior model is the weakness of the proposed mathematical framework based on such statistics. The elasticity matrix cannot appropriately simulate and model human behavior in response to price variations. Given that, unrealistic results are observed in Figure 11, emphasizing the need for further researches and developments over more practical methods.

\section{Conclusion}

To cope with the need for fast and flexible, but expensive, units in resilient renewable energy-based MGs, this paper augmented price-based demand response potentials in MG design problem in order to maximize profit of the MG owner. To ensure fairness of retail prices, regulatory constraints were considered in the model. Mainly to alleviate complexity of the proposed model, Benders decomposition technique was applied to decomposing the model into a master problem and operation and resiliency sub-problems. The master problem determined binary variables to optimally design an MG; the operation sub-problems optimized operation of the designed MG; and the resiliency sub-problem ensured safe operation of the MG during islanding event. Simulations for a sample MG illustrated efficiency and effectiveness of the proposed model and solution algorithm. Moreover, the results demonstrated a remarkable improvement in the profit of the MG owner as demand response potentials were enabled via the proposed approach.

\section{Nomenclature}

\section{Indices and symbols}

ch Superscript for energy storage charging mode

dch Superscript for energy storage discharging mode

flat Superscript for the situation without time-varying demand response program

I Index of DERs

$h, d, y \quad$ Indices of hour, period, and year

Sets

ES Set of batteries 
G

$S \quad$ Set of scenarios

$W \quad$ Set of non-dispatchable units

\section{Parameters}

E $\quad$ Elasticity coefficient matrix

$e \quad$ Elasticity coefficient

C Rated capacity of energy storage systems

c Generation price for dispatchable units

$C C \quad$ Annualized investment cost of generating units

CP Annualized investment cost of storage-power

$C E \quad$ Annualized investment cost of storage-energy

$K \quad$ Discount rate

$R \quad$ Revenue attained by the MG owner

$\eta \quad$ Energy storage efficiency

$w_{s} \quad$ Probability of scenario

$\rho \quad$ Market price

\section{Variables}

$D$

Demand after enabling demand response program

$P \quad$ Power output of DERs

$P_{M} \quad$ Power exchange between $\mathrm{MG}$ and the main grid

$U_{i, t} \quad$ State of charge $(0 / 1)$

$V_{i, t} \quad$ State of discharge $(0 / 1)$

$X \quad$ State of installing DERs (0/1)

$\lambda \quad$ Designed time-varying price

$\alpha a, \beta a, v, \quad$ Auxiliary variables
$\varphi, \theta, \chi$

\section{References}

1. "Critical infrastructure sectors", [Online]. Available: https://www.dhs.gov/cisa/critical-infrastructuresectors.

2. National Academy of Sciences, Enhancing the Resilience of the Nation's Electricity System, Washington, DC, USA (2017).

3. Guttromson, R. and Watson, J. "Defining, measuring, and improving resilience of electric power systems", In Smart Grid Handbook, pp. 1-21, John Wiley \& Sons, Ltd., Hoboken, New Jersey, USA (2016).

4. Field, C.B., Barros, V., Stocker, T.F., et al., IPCC (2012): Managing the Risks of Extreme Events and Disasters to Advance Climate Change Adaptation, Cambridge University Press, Cambridge, UK (2012).
5. Government Office of Science, Foresight Project of Reducing Risks of Future Disasters: Priorities for Decision Makers, Infrastructure and Resilience, London, UK (2012).

6. Khodai, A. "Guest editorial power grid resilience", IEEE Trans. Smart Grid, 7(6), pp. 2805-2806 (2016).

7. National Intelligence Council (NIC), Global Trends, Paradox of Progress, Washington, DC, USA (2017).

8. Governments of the United States and Canada, Joint United States-Canada Electric Grid Security and Resilience Strategy (2016).

9. Department of Energy, 2016-2020 Strategic Plan and Implementing Framework, Washington, DC, USA (2012).

10. Department of Energy, Los Alamos National Lab, Resilient Grid Operational Strategies, New Mexico, USA (2016).

11. Philips, J., Finster, M., and Pillon, J. "State energy resilience framework", REPORT ANL/GSS-16/4, Argonne National Lab., Chicago, Illinois, USA (Dec. 2016).

12. Potvin, J. and Short, T. "Distribution grid resiliency: overhead structures", REPORT 3002006780, EPRI, California, USA (2015).

13. Tripolitis, J., Martino S., and Rajagopalan, S. "Distribution grid resiliency: undergrounding", REPORT 3002006782, EPRI, California, USA (2015).

14. Department of Energy, Office of Energy Policy and Systems Analysis (EPSA), Resilience of the U.S. Electricity System: A Multi-Hazard Perspective, Washington, DC, USA (2016).

15. Anderson, J. and Deaver, B. "Distribution grid resiliency: modern grid technology", REPORT 3002006783, EPRI, California, USA (2015).

16. Clair, J. and Shahidehpour, M. "A functional microgrid for enhancing reliability, sustainability, and energy efficiency", Electric J., 25(8), pp. 21-28 (2012).

17. Lasseter, R.H. "Smart distribution: Coupled microgrids", Pro. of the IEEE, 99(6), pp. 1074-1082 (2011).

18. Shahidehpour, M. "Role of smart microgrid in a perfect power system", 2010 IEEE Power and Energy Society General Meeting (2010).

19. Strbac, G., Hatziargyriou, N., Lopes, J.P., et al. "Microgrids", IEEE Power and Energy Mag., 13(4), pp. 35-43 (2015).

20. Aguero, J.R., Bahramirad, S., and Khodaei, A. "Building resilient integrated grids", Electrification Mag., 3(1), pp. 48-55 (2015).

21. Bahramirad, S., Reder, W., and Khodaei, A. "Reliability-constrained optimal sizing of energy storages", IEEE Trans. Smart Grid, 3(4), pp. 2056-2062 (2012).

22. Alsaidan, I., Khodaei, A., and Gao, W. "A comprehensive battery energy storage optimal sizing model for microgrid applications", IEEE Trans. Power Syst., 33(4), pp. 3968-3980 (2018). 
23. Yuancheng, M. "Reliability and economy evaluation of microgrid based distribution network connection mode", 4th International Conf. on Electric Utility Deregulation and Restructuring and Power Technologies (DRPT), Shandong, China (2011).

24. Madathil, S.C., Yamangil, E., Nagarajan, H., et al. "Resilient off-grid microgrids: Capacity planning and N-1 security", IEEE Trans. Smart Grid, 9(6), pp. 6511-6521 (2018).

25. Billinton, R. "Maintaining supply reliability of small isolated power system using renewable energy", IEEE Proc. Generation, Transmission and Distribution, 148(6), pp. 530-534 (2001).

26. Arefifar, S.A. "Optimum microgrid design for enhancing reliability and supply-security", IEEE Trans. Smart Grid, 4(3), pp. 1567-1575 (2013).

27. Shahidehpour, M. and Khodaei, A. "Microgrid planning under uncertainty", IEEE Trans. Power Syst., 30(5), pp. 2417-2425 (2013).

28. Ranjbar, H. and Safdarian, A. "A robust model for daily operation of grid-connected microgrids during normal conditions", Sciantia Iranica J. (In Press). DOI:10.24200/sci.2019.50690.1819

29. Zhang, J. and Zeng, B. "Integrated planning for transition to low-carbon distribution system with renewable energy generation and demand response", IEEE Trans. Smart Grid, 29(3), pp. 1153-1165 (2014).

30. Yang, X. "Microgrid's generation expansion planning considering carbon economy", 2012 Asia-Pacific Power and Energy Engineering Conf., Shanghai, China (2012).

31. Shahidehpour, M. and Khodaei, A. "Microgrid-based co-optimization of generation and transmission planning in power systems", IEEE Trans. Power Syst., 28(2), pp. 1582-1590 (2013).

32. Cheng, B. and Wang, Z. "Robust optimization based optimal DG placement in microgrids", IEEE Trans. Smart Grid, 5(5), pp. 2173-2182 (2014).

33. Khodaei, A. "Resiliency-oriented microgrid optimal scheduling", IEEE Trans. Smart Grid, 5(4), pp. 15841591 (2014).

34. Khodaei, A. "Provisional microgrid planning", IEEE Trans. Smart Grid, 8(3), pp. 1584-1591 (2017).

35. Atia, R. and Yamada, N. "Sizing and analysis of renewable energy and battery systems in residential microgrids", IEEE Trans. Smart Grid, 7(3), pp. 12041213 (2016).

36. Wu, T., Yang, Q., Bao, X., et al. "Coordinated energy dispatching in microgrid with wind power generation and plug-in electric vehicles", IEEE Trans. Smart Grid, 14(1), pp. 47-57 (2013).

37. Zhang, C., Xu, Y., Dong, Z.Y., et al. "Robust coordination of distributed generation and price-based demand response in microgrids", IEEE Trans. Smart Grid, 5(4), pp. 1608-1620 (2017).

38. Ahmad Bashir, A., Pourakbari-Kasmaei, M., Contreras, J., et al. "A novel energy scheduling framework for reliable and economic operation of islanded and grid-connected microgrids", Electric Power Systems Research J., 171, pp. 85-96 (2019).

39. Safdarian, A. "Integration of price-based demand response in Discos' short-term decision model", IEEE Trans. Smart Grid, 5(5), pp. 2235-2245 (2014).

\section{Biographies}

Mohsen Chegnizadeh received his BSc from Amirkabir University of Technology, Tehran, Iran, in 2014, and the MSc degree from Sharif University of Technology, Tehran, Iran, in 2017, both in Electrical Engineering. His current research interests include power system resilience, smart grid issues, and distribution system operation and planning.

Amir Safdarian received the BSc degree from Tehran University, Tehran, Iran, in 2008, and the MSc and PhD degrees from Sharif University of Technology, Tehran, Iran, in 2010 and 2014, respectively. He is currently an Assistant Professor at Sharif University of Technology. His research interests include distribution system operation and planning, smart grid issues, and power system reliability and resilience. Dr. Safdarian was a recipient of the 2013 IEEE Power System Operation Transactions Prize Paper Award, 2016 IEEE Iran Section Best PhD Dissertation Award, and 2019 IEEE Iran Section Young Investigator Award. Also, he was on the list of outstanding reviewers of IEEE Transactions on Sustainable Energy in 2016. 